\title{
The Application of Thermodynamic Parameter Model Based on RGSA-RBFNN in Vacuum Resurgence Control Process
}

\author{
Xinye $\mathrm{Li}^{1}$, Yekuan Luo ${ }^{1}$, Jiajing Wang ${ }^{1}$ and Yushan $\mathrm{Hao}^{2}$ \\ ${ }^{1}$ School of Electrical and Electronic Engineering, North China Electric Power University, Baoding, China \\ ${ }^{2}$ Baoding Sanchuan Electrical limited liability company, Baoding, China
}

\begin{abstract}
In traditional vacuum resurgence control process, there are some problems such as insufficient moisture regain, unsatisfactory moisture content absorption and large steam loss. In order to solve these problems, we put forward the vacuum preconditioner's thermodynamic parameter model to control the vacuum resurgence process quantitatively. At first we proposed an Reinforcement Gravitational Search Algorithm (RGSA) to optimize the RBF neural network parameters, and then used the RGSA-RBFNN, GSA-RBFNN, RBFNN to establish the corresponding thermodynamic parameter model of the vacuum pre-conditioner. At last we used test data set to verify the model established by those three kinds of neural network. The results showed that RGSA-RBFNN has very strong function mapping ability, higher precision and more advantages than GSA-RBFNN or RBFNN. Using the model to control the process of vacuum resurgence, the moisture content of tobacco strips increases from $8 \%$ to $15 \%$, which is 4 percentage points higher than $11 \%$ of the unmodeled one. The moisture content of tobacco leaf has been improved greatly. It has certain guiding significance for tobacco strips production of vacuum pre-conditioner.
\end{abstract}

Keywords-vacuum resurgence; thermodynamic parameter model; RGSA; GSA; RBFNN

\section{INTRODUCTION}

Vacuum resurgence plays a key role in the process of tobacco leaf production. As the vacuumizing goes on, the pressure in vacuum pre-conditioner will be reduced continuously. When the pressure is reduced to a certain extent, we inject steam and water into the inner of cabinet for tobacco strips moisture regain. With the continuous injection of steam, there is a certain pressure and temperature difference between the interior and exterior of tobacco strips. The steam will permeate into tobacco strips under the differential pressure, which will liquefy and release heat. Thereby increasing the moisture content and temperature of the tobacco leaf $[1,2]$.

In actual production process, the cigarette factory mainly relies on the production experience, rather than giving a quantitative discriminant criteria in the process of vacuum resurgence. This lead to the insufficient moisture content absorption of tobacco strips, inadequate moisture regain, low loose rate, and so on. This phenomenon is not conducive to the follow-up process and affects the production quality at the same time. According to the literature consulted, there is few research on the parameters of water and vapor for vacuum pre- conditioner and few relative research on the model quantitative control of the vacuum resurgence process.

In this paper, we establish mathematical model of thermodynamic parameters (pressure, temperature, enthalpy, etc.) for vacuum pre-conditioner based on Reinforcement Gravitational Search Algorithm-RBFNN(RGSA-RBFNN), Gravitational Search Algorithm-RBFNN(GSA-RBFNN) and Radial Basis Function neural network (RBFNN). We can control how much vapor and water to add with the mathematical model. The quantitative control can be realized so as to improve the effect of vacuum resurgence, achieve the requirement of resurgence precision, and improve the quality of the tobacco production.

\section{PARAMETRIC RELATION OF VACUUM PRE-CONDITIONER AND CONTROL PRINCIPLE}

The vacuum pre-conditioner is mainly composed of a box, a vacuumizing system, a material transmission system and a vacuum resurgence system. When vacuum pump works, all valves are closed except for the evacuation valve. When the vacuumizing finished, the steam valve and water valve are opened for the vacuum resurgence of tobacco strips. After the moisture regain is completed, the broken valve will be opened and the vacuum resurgence of tobacco strips is completed.

\section{A. Relation of Thermodynamic Parameters}

The total energy of working substance includes internal energy and external energy, in engineering thermodynamics, enthalpy [3] is defined as:

$$
h=u+p v
$$

$u$ represents the thermodynamic energy of $1 \mathrm{~kg}$ working substance, which is the energy stored in $1 \mathrm{~kg}$ working substance. The pushing work of $1 \mathrm{~kg}$ working substance is $p v$.

The enthalpy of injection steam [3] is related to steam pressure and temperature, which can be expressed as:

$$
h_{\text {qout }}=f_{l}\left(P_{\text {qout }}, T_{\text {qout }}\right)
$$

$h_{\text {qout }}$ is the enthalpy of injection steam, $P_{\text {qout }}$ is steam pressure, and $T_{\text {qout }}$ is temperature. The internal steam enthalpy of the vacuum pre-conditioner is also a function of its temperature and pressure. It's relationship can be expressed as: 


$$
h_{\text {qin }}=f_{2}\left(P_{\text {qin }}, T_{\text {qin }}\right)
$$

$h_{\text {qin }}$ denotes the enthalpy of steam, $P_{\text {qin }}$ denotes steam pressure, and $T_{\text {qin }}$ denotes steam temperature in the vacuum pre-conditioner.

Temperature of vaporization point, $t_{\mathrm{d}}$, is a function of internal pressure in the machine. As the pressure decreases, temperature of vaporization point will also reduce. The temperature of vaporization point is $t_{\mathrm{d}}=100^{\circ} \mathrm{C}$ under $101.325 \mathrm{kPa}$, and the temperature is $t_{\mathrm{d}}=20.414^{\circ} \mathrm{C}$ under $2.4 \mathrm{kPa}$. The relation between the temperature of vaporization point and the steam pressure in the machine was established as follow:

$$
t_{\mathrm{d}}=f_{3}\left(P_{\text {qin }}\right)
$$

Replacing $T_{\text {qin }}$ with $t_{\mathrm{d}}$ in equation (3), the lowest enthalpy $h_{\text {low }}$ of the injection water vaporization in the machine is:

$$
h_{\text {low }}=f_{4}\left(P_{\text {qin }}, t_{\mathrm{d}}\right)
$$

\section{B. The Control Principle of Vacuum Resurgence Process}

Equation (3) minus equation (5) represents that the actual steam enthalpy in the machine minus the minimum steam enthalpy for water vaporization. The obtained value is the amount of enthalpy that needs to be increased or decreased. It is assumed that the flow rate and temperature of injection water are constant, which denotes that the enthalpy of injection water is constant. The injection enthalpy can be controlled by controlling the amount of injection steam.

(1) When $T_{\text {qin }}<t_{\mathrm{d}}$, the injection water at this time cannot be entirely vaporized, and the amount of injection steam should be increased to raise the temperature in the machine, so as to ensure that the water vaporize totally.

$$
h_{\text {low }}-h_{\text {qin }}=\Delta h_{\text {qout }}
$$

$\Delta h_{\text {qout }}$ denotes the amount of steam enthalpy that need to increased. So we can adjust the amount of injection steam quantitatively.

(2) When $T_{\mathrm{qin}}>t_{\mathrm{d}}$, too much steam enter the cabinet at this time, which will lead to serious steam waste. It is hoped to reduce the amount of steam, but it also make the injection water vaporize completely.

$$
h_{\text {qin }}-h_{\text {low }}=\Delta h_{\text {qout }}
$$

$\Delta h$ qout represents the amount of steam enthalpy that needs to be reduced, which adjust the amount of steam quantitatively.

(3) When $t_{\mathrm{d}} \approx T_{\text {qin }}$ and $t_{\mathrm{d}}$ is slightly less than $T_{\text {qin }}$, the injection water can be completely vaporized without wasting steam, keeping the amount of steam entering constantly at this time.

The functions $f_{1}, f_{2}, f_{3}, f_{4}$ are the models we need to establish. The above functional models are severally established by using RGSA-RBFNN, GSA-RBFNN, RBFNN. In the vacuum resurgence control process, the internal and external pressure, temperature of vacuum pre-conditioner can be read directly from the pressure, temperature instrument. By substituting the measured values into above models, the relevant thermodynamic parameters can be obtained and the vacuum resurgence control can be carried out according to equations (6) and (7).

\section{RGSA ALGORITHM AND RGSA-RBFNN MODELING}

There is an interaction force between any particle and there is a tendency to move towards a heavier particle. Inspired by Newton's law of universal gravitation and the law of motion, Esmat Rashedi, a professor at Iran's kerman university, and others proposed Gravitational Search Algorithm in the literature [4] in 2009. As we can know from [4 6], formula (8) is the force exerted by particle $j$ on particle $i$ at the moment $t$; formula (9) is the sum of forces exerted on particle $i$ from other particles at the moment $t$.

$$
\begin{gathered}
F_{i j}{ }^{k}(t)=G(t) \frac{M_{i}(t) \times M_{j}(t)}{R_{i j}(t)+\varepsilon}\left(x_{j}^{k}(t)-x_{i}^{k}(t)\right) \\
F_{i}{ }^{k}(t)=\sum_{j=1, j \neq i}^{N} \operatorname{rand}_{j} F_{i j}{ }^{k}(t)
\end{gathered}
$$

$M_{i}(t)$ and $M_{j}(t)$ represent the inertial mass of particle $i$ and particle $j$ severally and they relate to fitness function, $\varepsilon$ is a small constant, $G(t)$ is gravitational constant at moment $t$, $R_{i j}(t)$ is the Euclidean norm between particle $i$ and $j, \operatorname{rand}_{j}$ is a random number in the range $[0,1]$.

\section{A. Reinforcement Gravitational Search Algorithm}

In equation (8), it is obviously that the interparticle interaction force is opposite to interparticle distance. The greater distance is, the smaller force is, which makes the GSA degenerate into the random perturbation algorithm. On the contrary, the particle will vibrate at high speed near the optimal solution and the convergence rate will reduce. Because of these deficiencies, particle adjustable spacing $R_{i j}^{r(\psi)}$, inertial mass adjustment coefficient $\zeta$ and exponential adjustment weight $\eta$ are introduced in this paper. By adjusting particle spacing and inertial mass, the algorithm can improve its search ability and convergence speed to the optimal solution.

The variable $\psi(\psi \geq 0)$ is defined to describe the population density, it's expression is $\psi=\frac{1}{n} \sum_{i=1}^{n} \psi_{i}, n$ is the number of particles, and $\psi_{i}$ is the average distance between particle $i$ and other particles in the space. $\psi_{i}$ is shown in equation $(10), r(\psi)$ is defined in equation (11).

$$
\begin{gathered}
\psi_{i}=\frac{1}{n-1} \sum_{j=1, j \neq i}^{n} \sqrt{\sum_{k=1}^{D}\left(x_{j}^{k}-x_{i}^{k}\right)^{2}} \\
r(\psi)=\left\{\begin{array}{l}
r_{\text {min }}+\left(r_{\text {max }}-r_{\text {min }}\right) e^{1-1 / / /}, 0 \leq \psi<1 \\
r_{\text {min }}+\left(r_{\text {max }}-r_{\text {min }}\right) e^{1-\psi}, \psi \geq 1
\end{array}\right.
\end{gathered}
$$

$r_{\text {min }}$ and $r_{\text {max }}$ are the minimum and maximum values of index $R$ respectively. 
$M_{\zeta i}$ is the particulate inertial mass of $i$ after adjustment, described as equation (12).

$$
M_{\zeta i}=\left\{\begin{array}{l}
(1+\zeta)^{\eta} M_{i}(t), f i t_{i}(t)<f i t_{A V G}(t) \\
(1-\zeta)^{\eta} M_{i}(t), f i t_{i}(t)>f i t_{A V G}(t)
\end{array}\right.
$$

$f i t_{i}(t)$ and $\operatorname{fit}_{A V G}(t)$ are the fitness value and average fitness value of particle $i$ at time $t, \eta$ is the exponential adjustment weight, $\zeta \in[0,1)$. When $\eta>1$, the inertial mass distribution of spatial particles is wider, which enhances the local search ability of the algorithm. When $\eta<1$, the particle inertial mass distribution is more concentrated, and the global convergence of the algorithm is improved.

It is notable that the magnitude of gravity depends on $R_{i j}(t)$, $M_{j}, M_{i}$. Based on equations (8), (11) and (12), the force exerted by particle $j$ on particle $i$ at time $t$ can be expressed as:

$$
F_{i j R}^{k}(t)=G(t) \frac{M_{\zeta i}(t) \times M_{\zeta j}(t)}{R_{i j}^{r(\psi)}(t)+\varepsilon}\left(x_{j}^{k}(t)-x_{i}^{k}(t)\right)
$$

$\psi$ can adjust the distance between particles, $\zeta$ and $\eta$ can adjust the distribution of inertial mass effectively. We can adjust the size of $\psi, \zeta, \eta$ to balance the global and local search ability of the algorithm, which improve the convergence speed of the algorithm, so as to solve the problem that traditional GSA is easy to fall into local optimal solution.

The RGSA can be obtained by replacing equation (8) with equation (13) in literature [4], which can improve the search ability effectively by adjusting the interparticle distance and the inertial mass.

\section{B. Modeling with RGSA-RBFNN}

RBFNN [7,8] is a single hidden layer of three layer forward neural network. It is widely used in the nonlinear function approximation and the establishment of control system model. The parameters of RBF directly affect the performance of neural network. In this paper, an RGSA is proposed to optimize the parameters of RBFNN to obtain RGSA-RBFNN. The modeling process based on RGSA-RBFNN is shown in FIGURE I.

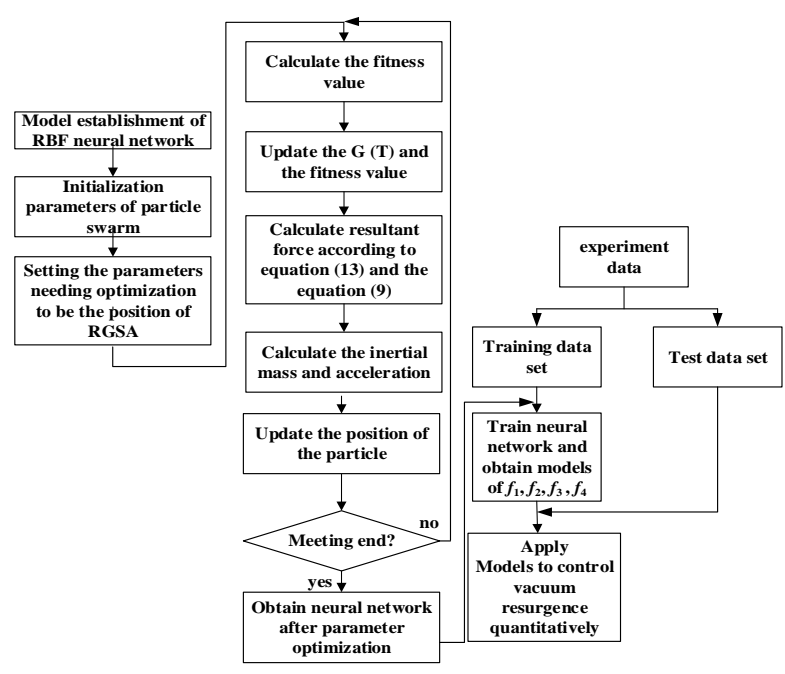

FIGURE I . FLOWCHART OF MODELING WITH RGSA-RBFNN

The literature $[9,10]$ apply GSA to optimize the parameters of RBFNN, which obtain effective results. Since GSA initialization is random, the inertial mass of particle and the particle spacing can not be adjusted, which makes GSA fall into local optimal solution easily. We introduced particle adjustable spacing, inertial mass adjustment coefficient in this paper, and proposed RGSA to optimize the parameters of RBFNN. RGSA successfully solves the problem that GSA fall into local optimal solution easily, improves the convergence speed of the algorithm and the training effect of neural network. According to the historical data of pressure and temperature meter of the steam pipe in a cigarette factory (the pressure is $1 \mathrm{MPa}$, the temperature is $200^{\circ} \mathrm{C}$ ), the training data set of $f_{1}$ has a pressure in the range of $0.8 \mathrm{MPa} \sim 1.2 \mathrm{MPa}$ and temperature in the range of $170 \sim 250^{\circ} \mathrm{C}$. According to the pressure and temperature inside vacuum pre-conditioner, the training data of $f_{2}, f_{3}, f_{4}$ is $200 \mathrm{~Pa} \sim 60 \mathrm{kPa}$ and $0 \sim 80^{\circ} \mathrm{C}$. The training data set contains three feature dimensions. We used training data set to train RGSA-RBFNN to obtain models $f_{1}, f_{2}$, $f_{3}, f_{4}$, and then applying those models to control the vacuum resurgence quantitatively.

\section{EXPERIMENT AND SIMULATION ANALYSIS}

We analysed the model $h_{\text {qout }}=f_{1}\left(P_{\text {qout }}, T_{\text {qout }}\right)$, as an example in this section, established by GSA-RBFNN, RGSA-RBFNN and RBFNN respectively. Test data set contains 310 data and it is different from the training data set, which pressure range from $0.955 \mathrm{MPa}$ to $1.123 \mathrm{MPa}$, and the corresponding temperature range from $180^{\circ} \mathrm{C}$ to $245^{\circ} \mathrm{C}$. The number of the training data set is shown in TABLE I.

TABLE I. THE NUMBER OF TRAINING DATA SET

\begin{tabular}{|c|c|c|c|c|c|c|}
\hline train & 1 & 2 & 3 & 4 & 5 & 6 \\
\hline No. & 448 & 896 & 2347 & 3787 & 5087 & 7673 \\
\hline
\end{tabular}

\section{A. The Model Simulation}

The data set train2 is used as a training set, the results of the test set under GSA-RBFNN, RGSA-RBFNN and RBFNN models are shown in FIGURE II. The percentage error represents the ratio of error to original vapor enthalpy. In the 
middle part, the test error of GSA-RBFNN and RGSA-RBFNN is smaller than RBFNN. The test effect of RGSA-RBFNN model is better than GSA-RBFNN and RBFNN model. Train5 is used as a training set, the results are shown in FIGURE III. The percentage error of RGSA-RBFNN model is zero nearly. The percentage error of GSA-RBFNN and RBFNN models are between $-0.03 \% \sim 0.05 \%$ and $-0.15 \% \sim 0.1 \%$ severally. Obviously, the training effect of RGSA-RBFNN model is better than the other two models.

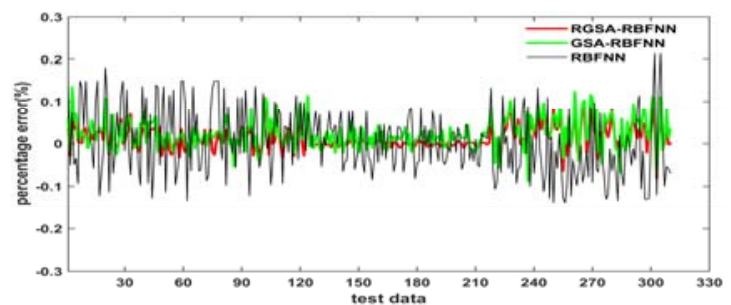

FIGURE II . MODEL RESULTS OF TRAIN2

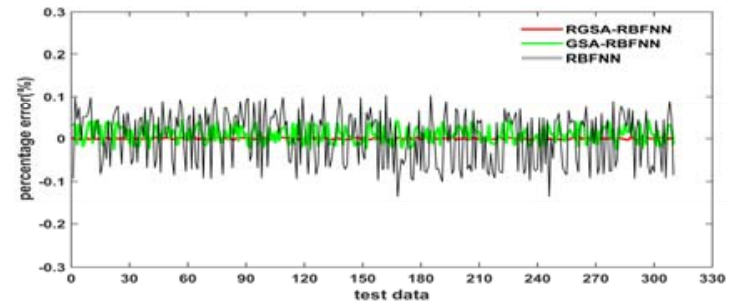

FIGURE III. MODEL RESULTS OF TRAIN

The corresponding test results with different training data set are shown in FIGURE IV. The mean-square error (MSE) is the quadratic sum of the error, divided by the number of test data, between the test results and original data. The error precision is the ratio of the mean error to the vapor enthalpy. The smaller the error precision is, the better the model is.
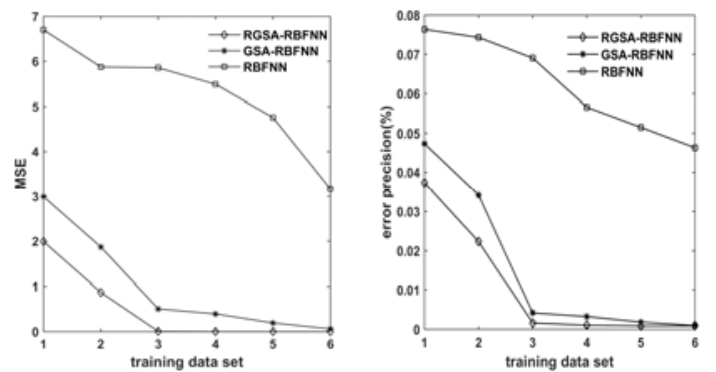

FIGURE IV. TEST RESULT CONTRAST DIAGRAM

From FIGURE IV, with the number increasing of training data, the MSE and error precision of training effect are decreasing gradually. The maximum, minimum MSE and error precision of RGSA-RBFNN are 1.99075, 0.00081, 0.03736\%, $0.00088 \%$. The effect of RGSA-RBFNN is better than GSARBFNN and RBFNN. From train3 to train6, the MSE and error precision of RGSA-RBFNN and GSA-RBFNN test results become small rapidly, the MSE and error precision of RBFNN test results are not obviously. RGSA-RBFNN test results are better than the other two kinds of neural network test results.

\section{B. Experiment Analysis}

The main task of vacuum resurgence is moisture regain. It is hoped that moisture content of tobacco strips will reach $20 \%$ after vacuum resurgence, but the moisture regain capacity of vacuum pre-conditioner in cigarette factory can only be increased $2 \% \sim 3 \%$. The electric coupling sensors are used and the response time of sensors is less than $2 \mathrm{~s}$. The real-time temperature and pressure collected by sensors substitute into models. According to the calculation results of modeling, the mensurable control of vacuum resurgence will carry out, which will improve the effect of moisture regain and the production quality of tobacco. According to the experiment collection data of moisture content, the moisture content of raw tobacco strips is quite different, and the highest and lowest moisture content is $8.92 \%$ and $7.95 \%$ respectively. Through the unmodeled vacuum pre-conditioner, the highest moisture content of tobacco strips is $11.07 \%$, and the moisture content of tobacco strips is not uniform. By establishing models to control the process of moisture regain, the moisture content is $15 \%$ and it is more uniform. The moisture content of tobacco increased 4 percentage points compared with the unmodeled one. The vacuum resurgence effect was significantly improved.

\section{CONCLUSION}

In this paper, we proposed RGSA to optimize the parameters of RBFNN, then we used RGSA-RBFNN, GSARBFNN, RBFNN to establish the aforementioned models respectively and we analyzed the injection steam modeling process detailedly. Test data set validating models showed that MSE and error precision of RGSA-RBFNN are better than GSA-RBFNN and RBFNN. The quantitative control of vacuum pre-conditioner is realized through the calculation results of thermodynamic parameter models. Through vacuum resurgence control process of vacuum pre-conditioner by modeling, the moisture content of tobacco strips increased from $8 \%$ to $15 \%$, which is 4 percentage points higher than the unmodeled one. The moisture regain is more sufficient and moisture content is increased obviously. Parameter model control is helpful to improve the production efficiency and quality of tobacco strips.

\section{ACKNOWLEDGEMENT}

Thanks for the support of Guidance plan project for the education department of Hebei province in 2012 (Z2012038).

\section{REFERENCES}

[1] Qinghua Ou, Wenbin Xu, Wen Wei, et al. Effect of vacuum humidity on the structure, chemical composition and esthetic quality of duplicate roasted tobacco leaf. Modern Food Science and Technology, 2009, 25(3): 260-261.

[2] Zhiming Pei, Juan Xu, Xiaobao Fan, et al. Design of and experiment on composite vacuum pre-conditioner.Tobacco Science \&Technology, 2016, 49(9): 89-92.

[3] Weidao Shen, Zhimin Jiang, Jungeng Tong. Engineering Thermodynamics. 3rd Edition[D]. Beijing: Higher Education Press, 2001, 27-36.

[4] Rashedi E, Nezamabadi-pour H, Saryazdi S.GSA: a gravitational search algorithm[J]. Information Sciences,2009,179(13):2232-2248.

[5] Bhattacharya A, Roy P K.Solution of multi-objective optimal power flow using gravitational search algorithm[J].Iet Generation Transmission \& Distribution, 2012, 6(8):751-763. 
[6] Li C, Zhang N, Lai X, et al. Design of a fractional-order PID controller for a pumped storage unit using a gravitational search algorithm based on the Cauchy and Gaussian mutation[J]. Information Sciences, 2017, 396:162-181.

[7] Guerra F A, Coelho L D S. Multi-step ahead nonlinear identification of Lorenz's chaotic system using radial basis neural network with learning by clustering and particle swarm optimization[J].Chaos Solitons \&Fractals,2008, 35(5):967-979.

[8] Schubert M, Muffler A, Mourad S. The use of a radial basis neural network and genetic algorithm for improving the efficiency of laccasemediated dye decolourization.[J]. Journal of Biotechnology, 2012, 161(4):429-436.

[9] Cao C, Song S, Chen J, et al. An Approach to Predict Debris Flow Average Velocity[J]. Water, 2017, 9(3):205.

[10] R. Ning Zhou L I, Feng X Y, Wei X J. Optimized Locomotive Adhesion Control Based on Dynamic Multiple Sub-group GSA-RBF Neural Network[J]. Journal of the China Railway Society, 2014, 36(12):27-34. 